\section{Use of Statistics as Another Factor Leading to an Overestimation of Chlorhexidine's Role in Skin Antisepsis}

To the Editor-We read with interest the article by Nuntnarumit and Sangsuksawang, ${ }^{1}$ describing a randomized clinical trial of $1 \%$ aqueous chlorhexidine ( $\mathrm{CHX}$ ) compared with $10 \%$ aqueous povidone-iodine (PI) for the prevention of blood culture contamination in neonates. We looked at the reported outcomes- 0 of 172 blood cultures contaminated in the CHX trial arm and 5 of 172 in the PI arm-for which the authors claim statistical significance in favor of $\mathrm{CHX}$. Apart from the unusual definition of blood culture contamination (including Staphylococcus aureus) and remaining uncertainty about whether aqueous or alcoholic CHX was used (due to differences between the text and Table 1), we noticed inconsistencies in the statistical analyses. The "Methods" section states that Fisher's exact test and the $\chi^{2}$ test were used. Our calculations, using 2-tailed $P$ values, show that the effect was nonsignificant by Fisher's exact test and the $\chi^{2}$ test with Yates's correction and significant only by the $\chi^{2}$ test without Yates's correction. The use of one-tailed $P$ values would be inappropriate, because this would assume a priori that only $\mathrm{CHX}$ can be superior. Although there is some debate about Yates's correction, standard references recommend using it when at least one of the expected cell counts is below $5,{ }^{2,3}$ which is the situation here. That means that 2 out of the 3 applicable tests, including Fisher's exact test, show nonsignificant results. We believe that the reporting was not fully transparent and that the authors should have indicated that not all test variants listed in "Methods" gave significant results. Furthermore, the study combined data from both catheter-drawn and skindrawn blood cultures. It is questionable whether a catheterdrawn blood culture adequately reflects the effect of skin antisepsis, and the process of drawing through a catheter is biologically so different that it may not be sensible to combine data from these 2 methods of blood sampling.

With the present example, ${ }^{1}$ there are now 3 factors in the published literature that contribute to an overestimation of CHX's role in skin antisepsis: (1) a failure to adequately neutralize antiseptics in microbiological tests, (2) a mistaken attribution of clinical trial outcomes from $\mathrm{CHX}$-alcohol combinations to CHX alone, and (3) inconsistent or nontransparent use of statistics.

First, it is a basic requirement in both US and European standardized microbiological efficacy testing of antiseptics to use adequately validated neutralizer substances in the sampling medium, in order to prevent continued killing of microorganisms after sampling has taken place. Although in principle this applies to all antiseptics, it is of particular rel- evance to $\mathrm{CHX}$, because of documented false-positive efficacy assessments in the absence of neutralizers or with inadequate ones. ${ }^{4-6}$ Many published microbiological studies and clinical trials that report microbial skin counts lack adequate information about neutralizer use, making their results open to question. Second, prompted by irregularities in published articles, a recent systematic review ${ }^{7}$ found that trial outcomes in the areas of skin antisepsis for blood culture collection, vascular catheter insertion, and surgical skin preparation were often incorrectly attributed solely to $\mathrm{CHX}$ when, in fact, $\mathrm{CHX}$-alcohol combinations had been used. This affected a range of $29 \%-43 \%$ (between the 3 applications) of clinical trials and systematic reviews and led to unsubstantiated recommendations in prominent clinical-practice guidelines. This has resulted in a widespread belief in the efficacy of $\mathrm{CHX}$ in the infection control community that was in significant parts actually based on evidence for the efficacy of the combination of CHX and alcohol. Remarkably, only about $35 \%$ of clinical trials and systematic reviews both (1) correctly listed the antiseptics that were trialed and their active ingredients and (2) correctly attributed trial outcomes to the actual antiseptics tested. Third, the use of statistics in favor of $\mathrm{CHX}$ is evident in another example. A well-known trial of skin antiseptics for vascular catheter insertion and maintenance ${ }^{8}$ used 3 antiseptics-CHX alone, isopropanol (IPA) alone, and PI alone-in a parallel 3-arm trial. When comparing $\mathrm{CHX}$ alone against the other 2 groups separately (for the outcome catheter colonization), we found that only the single difference between CHX and PI was significant; the difference between $\mathrm{CHX}$ and IPA was not. However, the authors concluded that CHX was superior to IPA and PI, on the basis of analyses that combined the outcome data from IPA and PI into a single composite group and compared it against $\mathrm{CHX}$ alone. A clear rationale for combining the two was not provided. Although a table footnote also mentions an adjustment for different numbers of arterial and venous catheters in trial arms-which may yield significance, depending on further analyses-there is no mention that the primary numerical outcomes fail to show a significant difference between IPA and CHX. This article has been cited in multiple recommendations for CHX for skin antisepsis.

What does this all mean? Of course, it does not mean that $\mathrm{CHX}$ is not superior to alternative substances for some applications, but it does highlight that the performance of different skin antiseptics has been inappropriately judged on multiple occasions, often in favor of CHX. Thus, it would be wise for the infection control community to reevaluate the role and the usefulness of $\mathrm{CHX}$ and other compounds for skin antisepsis. Ideally, this should be done with a broader, more holistic approach, incorporating (1) insight from microbiological testing, (2) outcomes from clinical trials, (3) 
appropriate historical information, and (4) the principle of biological plausibility. ${ }^{9}$
February 7, 2013. http://webbertraining.com/recordingslibraryc4 .php. Published 2013. Accessed March 27, 2013.

\section{ACKNOWLEDGMENTS}

Potential conflicts of interest. All authors report no conflicts of interest relevant to this article. All authors submitted the ICMJE Form for Disclosure of Potential Conflicts of Interest, and the conflicts that the editors consider relevant to this article are disclosed here.

Matthias Maiwald, MD, PhD; ${ }^{1}$ Trevor N. Petney, $\mathrm{PhD}^{2}$
Pryseley N. Assam, ${ }^{2}$

Affiliations: 1. Department of Pathology and Laboratory Medicine, KK Women's and Children's Hospital, Singapore; Department of Microbiology, National University of Singapore; and Duke-National University of Singapore Graduate Medical School, Singapore; 2. Department of Ecology and Parasitology, Zoology Institute, Karlsruhe Institute of Technology, Karlsruhe, Germany; 3. Centre for Quantitative Medicine, Office of Clinical Sciences, Duke-National University of Singapore Graduate Medical School, Singapore; Department of Epidemiology, Singapore Clinical Research Institute, Singapore; and Singapore Branch, Australasian Cochrane Centre, Singapore.

Address correspondence to Matthias Maiwald, $\mathrm{MD}, \mathrm{PhD}$, Department of Pathology and Laboratory Medicine, KK Women's and Children's Hospital, 100 Bukit Timah Road, Singapore 229899, Singapore (matthias.maiwald @kkh.com.sg).

Infect Control Hosp Epidemiol 2013;34(8):872-873

(C) 2013 by The Society for Healthcare Epidemiology of America. All rights reserved. 0899-823X/2013/3408-0023\$15.00. DOI: 10.1086/671282

\section{REFERENCES}

1. Nuntnarumit $P$, Sangsuksawang N. A randomized controlled trial of $1 \%$ aqueous chlorhexidine gluconate compared with $10 \%$ povidone-iodine for topical antiseptic in neonates: effects on blood culture contamination rates. Infect Control Hosp Epidemiol 2013;34(4):430-432.

2. Agresti A. An introduction to categorical data analysis. 2nd ed. Hoboken, NJ: Wiley, 2007.

3. Zar JH. Biostatistical analysis. 5th ed. Upper Saddle River, NJ: Pearson Prentice-Hall, 2010.

4. Kampf G, Shaffer M, Hunte C. Insufficient neutralization in testing a chlorhexidine-containing ethanol-based hand rub can result in a false positive efficacy assessment. BMC Infect Dis 2005;5:48.

5. Rittle KH. Efficacy of surgical preparation solutions in foot and ankle surgery. J Bone Joint Surg Am 2006;88(5):1160-1161.

6. Kampf G, Reichel M, Hollingsworth A, Bashir M. Efficacy of surgical hand scrub products based on chlorhexidine is largely overestimated without neutralizing agents in the sampling fluid. Am J Infect Control 2013;41(1):e1-e5.

7. Maiwald M, Chan ES. The forgotten role of alcohol: a systematic review and meta-analysis of the clinical efficacy and perceived role of chlorhexidine in skin antisepsis. PLOS ONE 2012;7(9): e44277.

8. Maki DG, Ringer M, Alvarado CI. Prospective randomised trial of povidone-iodine, alcohol, and chlorhexidine for prevention of infection associated with central venous and arterial catheters. Lancet 1991;338(8763):339-343.

9. Maiwald M. Why evidence should have biological plausibility: the story of chlorhexidine and its role in skin antisepsis. Teleclass,

\section{Reply to Maiwald et al}

To the Editor-We really appreciate the comments of Maiwald et al. ${ }^{1}$ on our article ${ }^{2}$ and would like to respond as follows.

1. The chlorhexidine (CHG) used in our study was in aqueous solution to make sure that the antiseptic property was solely of $\mathrm{CHG}$, not of alcohol, and to assess contact dermatitis secondary to CHG as well. CHG in alcohol solution has been reported to increase the risk of skin irritation, which may be related to alcohol itself irritating the skin or to $\mathrm{CHG}$ inducing a reaction or hypersensitivity.

2. We included Staphylococcus aureus as a contamination because most infants (330/344 [96\%]) in our study had blood cultures drawn on day 1 (on admission) to rule out infection. Skin flora organisms, that is, coagulase-negative staphylococci, or S. aureus, are very unlikely to be the cause of earlyonset sepsis in newborns. For late-onset sepsis, $S$. aureus is one of the most common organisms, especially in neonates with a central venous catheter; however, we would consider this organism as a cause of infection on the basis of clinical circumstance, and if this is the case, antibiotics would be continued with an adequate duration, usually for at least 7 days. This is the reason we defined culture contamination in such a way that antibiotics have to have been discontinued before 3 days, together with clinical improvement.

3. We included blood cultures taken from umbilical catheters because umbilical catheterization (UC) is a very common procedure in neonates on admission and because the UC procedure is not like other central line access, as steps of procedure itself, including tapping, holding, and cutting the cord, are prone to contamination. There is also a chance of the skin antiseptic preparation not being followed correctly, for example, inadequate time to let antiseptic dry. We have seen blood culture contamination from UC on admission in our unit from time to time.

4. Regarding the statistical issue, we appreciate the comments. As mentioned in the "Discussion" of the original article, ${ }^{2}$ because the incidence of blood culture contamination was lower than we expected, it is possible that the null hypothesis would not have been rejected if more infants had been enrolled.

\section{ACKNOWLEDGMENTS}

Potential conflicts of interest. All authors report no conflicts of interest relevant to this article. All authors submitted the ICMJE Form for Disclosure of Potential Conflicts of Interest, and the conflicts that the editors consider relevant to this article are disclosed here. 